\title{
Crisis de la Educación Superior en el Chile neoliberal: mercado y burocracia
}

\section{Crise da Educação Superior no Chile neoliberal: mercado e burocracia}

\section{Crisis of Higher Education in neoliberal Chile: market and bureaucracy}

\author{
Nicolas Gregorio Fleet Oyarce* \\ Peodair Seamus Leihy** \\ Jose Miguel Salazar Zegers ${ }^{* * *}$
}

\begin{abstract}
RESUMEN
Este artículo analiza los conflictos y dilemas de la Educación Superior chilena, construida y masificada bajo políticas neoliberales desde 1980 hasta nuestros días. En particular, el trabajo se centra en los efectos de la reforma impulsada por el gobierno de Michelle Bachelet en 2016, la que tuvo como objetivo declarado quitar centralidad al mercado y garantizar la gratuidad de los estudios superiores, intentando con ello responder al movimiento social en 2011, protagonista del mayor ciclo de protestas conocido desde el retorno a la democracia en 1990 en Chile hasta ese momento. Basado en un análisis socio-histórico, con énfasis en el análisis de la transformación del Estado, se concluye que la Educación Superior mantiene su inercia mercantil, blindada por una burocracia estatal de estrecho vínculo con los poderes de mercado e ideológicos que controlan la enseñanza superior privada. En este arreglo, la crisis generada por la expansión de mercado -de legitimidad de la Educación Superior y sentido de su proyecto- sigue irresuelta.

Palabras clave: Educación Superior. Neoliberalismo. Burocracia. Bachelet. Gratuidad.

* Universidad Católica Silva Henríquez. Santiago, Chile. E-mail: nicholas.fleet@gmail.com http://orcid.org/0000-0002-7570-2715

*** Pontificia Universidad Católica de Chile. Santiago, Chile. E-mail: peleihy@uc.cl - http:// orcid.org/0000-0002-5569-0905

*** Universidad de Valparaíso. Valparaíso, Chile. E-mail: jose.salazar@uv.cl - http://orcid.
\end{abstract} org/0000-0003-4636-1779 


\title{
RESUMO
}

Este artigo analisa os conflitos e dilemas da Educação Superior chilena, construída e massificada pelas políticas neoliberais de 1980 até os dias atuais. Em particular, o trabalho centra-se nos efeitos da reforma promovida pelo governo de Michelle Bachelet em 2016, que tinha o objetivo declarado de retirar a centralidade do mercado e garantir o ensino superior gratuito, procurando assim responder ao movimento social no. 2011, protagonista do maior ciclo de protestos conhecido desde o retorno à democracia em 1990 no Chile até então. A partir de uma análise sócio-histórica, com destaque para a análise da transformação do Estado, conclui-se que a Educação Superior mantém sua inércia comercial, resguardada por uma burocracia estatal intimamente ligada ao mercado e aos poderes ideológicos que controlam a Educação Superior. privado. Nesse arranjo, a crise gerada pela expansão do mercado - da legitimidade da Educação Superior e do sentido de seu projeto - permanece sem solução.

Palavras-chave: Ensino Superior. Neoliberalismo. Burocracia. Bachelet. Gratuidade.

\begin{abstract}
This article analyzes conflicts and dilemmas within Chilean Higher Education, built and massified as it was under neoliberal policies from 1980. In particular, the work focuses on the effects of reforms presented by the government of Michelle Bachelet in 2016, with the stated objective of removing the centrality of market mechanisms and guaranteeing access to Higher Education. These reforms sought respond to 2011's boiling over of social protests, then the most intense since Chile's return to democracy in 1990. Based on socio-historical analysis, with emphasis on the transformation of the state's role, Higher Education is seen to maintain its market inertia, entrenched by a state bureaucracy with close ties to ideological powers that control Higher Education. private. Under this arrangement, the crisis generated by the expansion of the market -as to the legitimacy of Higher Education and the coherence of its project- remains unresolved.

Keywords: Higher Education. Neoliberalism. Bureaucracy. Bachelet. Free education.
\end{abstract}




\section{Introducción}

Chile ha experimentado en las últimas décadas un acelerado proceso de masificación de su Educación Superior. Desde una cobertura bruta de 15\% en 1990 -la misma de 1973 al inicio de la dictadura de Pinochet- se ha llegado en 2017 a $73 \%{ }^{1}$. Un rasgo particular de este proceso es que, producto del modelo neoliberal implantado en los años ochenta, la masificación ha corrido a cargo del sector privado bajo un arreglo de mercado.

Como se sabe, el neoliberalismo promueve a la Educación Superior como un espacio de movilidad social, haciendo de la aspiración a ascender individualmente un horizonte dominante que reemplaza las viejas lógicas colectivas de redistribución del Estado de Compromiso. En Chile, bajo gobiernos democráticos, la expansión educativa de nivel terciario fue impulsada precisamente en esta clave (GUTIÉRREZ; LÓPEZ; RUÍZ SCHNEIDER, 2018). Tanto para el Estado como para las instituciones y los individuos, la promesa de movilidad social por la vía de la Educación Superior fue determinante para dar sustento y legitimidad al Chile de la transición a la democracia (GUTIÉRREZ; LÓPEZ; RUÍZ SCHNEIDER, 2018). Este arreglo exhibió sus contradicciones y límites en el movimiento social de 2011. Una protesta estudiantil de gran envergadura, que por primera vez unificó a los estudiantes del sector tradicional -previo al neoliberalismo- y del nuevo segmento privado de mercado; criticó los efectos de la masificación mercantil y los límites del ascenso social prometido. Con demandas y consignas críticas del modelo neoliberal, los estudiantes chilenos sintetizaron su horizonte en la consigna Educación pública, gratuita $y$ de calidad.

En la medida que el propio régimen había trasladado a la educación la responsabilidad de la igualdad por su papel de agente de movilidad social, la desigualdad del neoliberalismo -una fuerte concentración de la riqueza- aparecía como problema de la educación. La herida educativa se proyectaría, entonces, como una crisis de legitimidad tanto de la educación como de la estructura de oportunidades de la sociedad chilena en general.

De 2011 a nuestros días la Educación Superior sufriría dos grandes procesos de cambio. El primero tiene que ver con la ralentización de su crecimiento y posterior consolidación de su matrícula de 2015 hasta hoy. El segundo cambio es

1 Elaboración propia a partir de datos reales de matrícula de 2017 del Sistema de Información de la Educación Superior del Ministerio de Educación de Chile (SIES) Disponible en: www.mifuturo. cl, y estimación en base a la Encuesta de Caracterización Socioeconómica (CASEN) también de 2017, última edición disponible. 
el conjunto de modificaciones legales que experimentó en la reforma impulsada por el segundo gobierno de Michelle Bachelet. La reforma educativa fue el eje de su programa, y en gran medida el tópico más problemático y polémico del debate nacional entonces. Bachelet intentó expresar las consignas del movimiento de 2011 en modificaciones institucionales, introduciendo un financiamiento por gratuidad a una parte de la población atendida -aproximadamente un tercio- e incrementado las exigencias a las instituciones, lo que iría desde una mayor estandarización en el aseguramiento de la calidad a la proscripción formal del lucro en ciertos casos.

Pero a pesar de la nueva institucionalidad, del incremento del gasto público y de la instauración de la subvención de gratuidad, la crisis de legitimidad de la Educación Superior no ha sido superada. Se podría decir, de hecho, que ha ocurrido todo lo contrario. Los cuestionamientos a la educación aumentan y, aparte, las críticas a la desigualdad de la sociedad chilena -y a la ilegitimidad de la misma- han derivado en demostraciones sociales cada vez más notorias. El estallido social de octubre de 2019 es, entonces, resultado de un largo proceso de acumulación de malestares, desnudando la incapacidad de las instituciones políticas para procesarle.

El presente trabajo intenta una revisión de la crisis de legitimidad de la Educación Superior chilena tras su masificación de mercado y posterior consolidación de tamaño. Sigue a esta introducción una contextualización socio-histórica del problema de la Educación Superior y del juego de actores que se conformó en su conflicto en los últimos años. Luego profundizamos en un aspecto clave de este trance: la acción estatal. En este apartado abordamos con detalle cómo se desarrolla un Estado neoliberal y qué tipo de prácticas instala. Un breve apartado de discusión cierra el artículo.

\section{La Educación Superior neoliberal: masificación y desborde}

Así como el Estado neoliberal se construye con la represión a los trabajadores organizados (O’DONNELL, 1978) y la institucionalización del dominio del capitalismo financiero en el centro de su poder político (SILVA, 1996), el desarrollo de la Educación Superior en este marco se ve precisamente determinado por la convergencia de estos dos tipos de intereses, es decir, de un proyecto conservador (SALAZAR; LEIHY, 2013) y otro de mercado (MONCKEBERG, 2005). Buena parte de esta síntesis queda expresada en el carácter subsidiario del Estado neoliberal, que renuncia a un papel directo en 
la economía y la sociedad y se limita a apoyar y subsidiar el desenvolvimiento de los privados (ORELLANA, 2012).

En este contexto durante los años ochenta, en plena dictadura militar, la Educación Superior neoliberal emerge, por un lado, de la reacción al proceso de Reforma Universitaria anterior (1967-1973), que buscaba expandir la enseñanza terciaria y alinearla con el proceso de modernización que experimentaba la sociedad chilena; y por el otro, la Educación Superior se privatiza para generar mercado:el financiamiento de las universidades públicas se reduce, los aranceles son pagados por los estudiantes, y nuevas instituciones privadas ofrecen un boyante mercado de opciones (BRUNNER, 1987). Mientras el Estado subsidia el desarrollo de este mercado, el sector público restringe su alcance para favorecer la expansión privada (ORELLANA, 2012). Como se puede leer en la opinión de Bardón, uno de los economistas precursores del modelo neoliberal, la contrarreforma se justifica en función de la mencionada constelación de intereses conservadores y de mercado.

Lo más notable... es que en nuestro país el Estado ayuda a la organización de los agitadores. En lugar de dejar en libertad a los estudiantes para organizarse como quieran, les dicta normas para que se junten y los obliga a afiliarse. ¿Por qué no se aplica la lógica de libertad del Plan Laboral? ¿Por qué no se aplica la lógica económica más elemental a la educación superior? (BARDÓN, 1980 en RUÍZ-SCHNEIDER, 2007, p. 104).

El plan laboral referido es el Código del Trabajo de 1979, que prohibió el activismo político en sindicatos y los limitó la articulación de huelgas más allá de una firma. El mismo efecto fue buscado para la Educación Superior. La contrarreforma neoliberal buscó entonces eliminar las condiciones del sistema universitario - tales como su carácter público, expansivo, gratuito y politizado- que favorecieron la articulación del movimiento estudiantil de los años sesenta y setenta, y más ampliamente, la politización de la sociedad y de su esfera intelectual.

La política neoliberal, junto con desfinanciar y restringir el crecimiento de la Educación Superior pública (BRUNNER, 1982), dispone de incentivos para que las nuevas instituciones privadas, universitarias o no universitarias (SALAZAR; LEIHY, 2013), lideren el nuevo ciclo de expansión del sistema. En estas condiciones, se anticipaba que el Movimiento Estudiantil (con mayúsculas), tal como representó la Reforma Universitaria, moriría y sería sustituido por una pluralidad de movimientos estudiantiles (con minúscula) que, sobre la base de 
la diferenciación competitiva del mercado, expresarían una superestructura fragmentada de grupos de interés (BRUNNER, 1985).

En la medida que el Estado chileno adopta un discurso y un esquema de financiamiento para impulsar la masificación mercantil de la Educación Superior, en 2011 este proceso llega ad portas del acceso universal (TROW, 2007). Según datos del Ministerio de Educación, en 2011, las instituciones privadas nuevas representan $77 \%$ de la matrícula total. Mientras el sistema en su conjunto creció en una tasa anual del $7 \%$ desde 1990 , las universidades privadas nuevas crecieron en $15 \%$ y las universidades tradicionales en $5 \%$.

Podemos distinguir una dimensión estructural y una ideológica de este proceso. Desde la estructura, se evidencia una transición subyacente hacia la generalización del trabajo intelectual en una economía postindustrial de servicios. Si bien el caso chileno no es necesariamente ejemplar de este proceso, la expansión de las burocracias de servicio constituye el rasgo más marcado del cambio en su estructura social (RUIZ, 2013; TORCHE; WORMALD, 2004). Asimismo, la ampliación del Estado neoliberal también está alineada con la masificación de la Educación Superior (FLEET, 2019). Una nueva "intelectualidad masiva" aparece internamente diferenciada en función de la segmentación de la Educación Superior sobre la base de preexistentes jerarquías de clases (VERCELLONE, 2007). En consecuencia, la mayoría de este grupo proviene de "nuevas generaciones" que se incorporan al trabajo intelectual a través de la expansión del sector masivo, privado, lucrativo y de menor calidad. Mientras en 2011 el 69\% de los estudiantes eran primera generación en la Educación Superior, 63\% de los hijos de trabajadores y empleados no calificados que estaban matriculados en ella asistían a instituciones no selectivas (ORELLANA, 2011).

En el ámbito ideológico, la masificación de la Educación Superior modela una cultura del trabajo inmaterial. Ideales profesionales, de ascenso social y de consumo circulan en la publicidad de la Educación Superior (SIMBÜRGER, 2013). Característicamente en Chile, las universidades - segmentadas, como vimos, según el origen de clase de los estudiantes - también se afirman como aparatos ideológicos, constituyendo sujetos profesionales para vocaciones y roles específicos en la superestructura. Por lo tanto, el neoliberalismo también segmenta orientaciones y sentidos que son marcadamente ideológicos en la medida que se vinculan con bases sociales específicas (FLEET, 2019) y con marcadas visiones político-ideológicas, según el dueño o régimen en que las instituciones operan (MONCKEBERG, 2007).

La culminación de este ciclo expansivo marca la crisis del modelo neoliberal en Educación Superior. Si bien las condiciones de masificación segmentada, tanto material como ideológica, así como la competencia de 
mercado y la privatización del costo de la educación, previnieron por décadas la articulación del movimiento estudiantil, tal como se esperaba del diseño original; en 2011 las mismas condiciones terminan propiciándolo.

Mientras en 2006 el movimiento de estudiantes secundarios puso de manifiesto la desigualdad en el acceso a la Educación Superior (DONOSO, 2013), en 2011 el movimiento irrumpe dentro del sistema universitario. Sobre la base del endeudamiento acumulado por la expansión del sistema, problemas típicos de funcionamiento - a saber, falta de becas en la Universidad de Chile y el intento de venta de la privada Universidad Central - terminaron por articular la protesta. El caso de la Universidad Central es, en todo caso, característico. Pertenece al pequeño grupo de universidades privadas masivas que no tienen fin de lucro o que comparten una orientación social, en conformidad con los intereses de sus sostenedores: organizaciones académicas, ONGs y congregaciones más progresistas de la Iglesia (Jesuitas y Salesianos). Se diferencian, de esta manera, de las universidades privadas masivas comerciales, en manos de corporaciones y multinacionales, y también de las universidades privadas de elite (conocidas como "cota mil" por estar en las faldas de la precordillera en Santiago) que cumplen un rol más ideológico formando liderazgo para los actores a los que se vinculan: empresariado, partidos políticos de derecha y órdenes conservadoras de la Iglesia (Opus Dei y Legionarios de Cristo) (MONCKEBERG, 2007).

Al oponerse a la venta de la Universidad Central, el movimiento se orientó a la recuperación (del sentido) de la universidad pública, excediendo el marco de las universidades del Estado en conformidad se amplía la base del movimiento. En tanto, la demanda por educación gratuita (el lema del movimiento fue "educación pública, gratuita y de calidad") se orienta hacia la recuperación del trabajo de la explotación del sistema (vía endeudamiento) y de su sentido público (liberado de la presión de ser recuperado como inversión privada) $(\mathrm{CONFECH}$, 2011). Con todo, a pesar de que el leitmotiv del movimiento estudiantil fue la oposición al lucro en Educación Superior, la movilización no provino de los estudiantes del sector lucrativo del sistema, sino que principalmente de las universidades tradicionales, históricamente organizadas a través de la Confederación de Estudiantes de Chile (CONFECH), y de las universidades privadas de orientación social (FLEET; GUZMÁN-CONCHA, 2017).

Las demandas por reforma del sistema de Educación Superior fueron integradas por el programa que llevó a Michelle Bachelet (BACHELET, 2013) nuevamente al poder en 2014, de la mano de la coalición de Tercera Vía que gobernara el país de 1990 hasta 2010, la ex Concertación de Partidos por la Democracia, ahora llamada Nueva Mayoría por la inclusión del Partido Comunista. En efecto, en dicho programa se comprometió la gratuidad en educación, junto con una reforma tributaria que la financiara y una nueva 
Constitución que también avanzara un enfoque de derechos sociales. Qué tanto este paquete contribuyó o no a cambiar la dirección del Estado es objeto aún de polémica -lo que abordamos adelante-, pero lo cierto es que coincide y también incide en una ralentización del crecimiento y consolidación de la Educación Superior chilena.

En 2011 el crecimiento del sistema encuentra un punto de inflexión contrayendo su expansión al 2\% anual hasta 2019. La contracción se concentra en las universidades privadas masivas comerciales, otrora el sector más expansivo: desde 2008 a 2011 ya venían creciendo en $11 \%$ anual y entre 2011 y 2019 su curva "se aplana" al 0,04\%. Mientras las universidades públicas tradicionales reducen su crecimiento por debajo de los límites que el régimen de gratuidad le impone, las universidades privadas de elite ("cota mil") lideran el crecimiento proporcional, con $4,2 \%$ anual.

Con la Reforma, el neoliberalismo de la Educación Superior pierde vectores cruciales, como la capacidad del mercado para determinar, por sí mismo, la asignación de los recursos y la calidad de las instituciones, así como el sentido de responsabilización individual por los costos de la educación como base motivacional del compromiso con los estudios -contra otras formas de compromiso político o social. No obstante, el marco neoliberal permanece como orientación general del sistema: instituciones siguen compitiendo por reclutar a estudiantes portadores de la gratuidad/voucher y el Estado subsidia un sistema que, aún privatizado y segmentado, es ciego con respecto a su sentido público.

Pero la crítica del movimiento estudiantil, y los derechos institucionalizados con la Reforma, abren un camino para la crítica del Estado neoliberal en su conjunto. En este sentido, la contradicción entre el reconocimiento de derechos sociales y el marco neoliberal del Estado, como se expresa en esta Reforma de la Educación Superior, no permiten recuperar la legitimidad de la dominación política, y más bien lo que se produce es una generalización de la protesta. En octubre de 2019, un estallido social de proporciones históricas lleva la crítica sectorial iniciada con el movimiento estudiantil a una crisis general del Estado. Aunque no es liderada por los estudiantes, la intelectualidad masiva que emerge de la masificación y politización de la Educación Superior en Chile sigue representando la mayoría a la base social y de soporte ideológico de la protesta (NÚCLEO DE SOCIOLOGÍA CONTINGENTE, 2020). 


\section{El Estado neoliberal de segunda generaciónen Educación Superior}

Uno de los aspectos más confusos en el debate sobre privatización educativa es el que dice relación con la supuesta reducción del Estado. Aunque es una máxima muy divulgada, lo cierto es que el proceso descrito arriba de masificación hubiese resultado imposible sin el concurso del Estado. La Reforma de 2016 converge con el agotamiento de la segunda ola de masificación de la matrícula, lo que a la postre es decisivo para entender aspectos claves de la morfología actual de la Educación Superior. El Estado chileno crece y hace un enorme esfuerzo de reorganización del desarrollo progresivo de instrumentos de política y mecanismos de coordinación sectorial. Pero este esfuerzo no concluye en un marco general que pudiera guiar y coordinar la evolución del sector, ni aún con el apoyo permanente del Banco Mundial (SALAZAR; RIFO,2020).

Aunque parecen largamente internalizadas dentro del discurso público y especializado, las nociones de calidad y equidad se incorporan a las políticas chilenas de Educación Superior hacia 1997, a propósito de una nueva política sectorial. De hecho, en el diseño original, el tema de la calidad de la Educación Superior solo aparece como consecuencia de la competencia de mercado. Solo después del fin de la dictadura se comienzan a incorporar marcos regulatorios dentro de las llamadas reformas de segunda generación del Estado neoliberal.

Quizás por el claro carácter aspiracional de las máximas de equidad y calidad, ambos términos han sido retóricamente explotados en el desarrollo de las políticas públicas. No obstante, aquí poseen una connotación más bien precisa: mientras calidad se asocia a la medición y el control (y permite justificar la instalación de sistemas de gestión empresarial y la emergencia de evaluaciones externas a las universidades), equidad refiere a la necesidad de cuidar que los segmentos sociales más pobres sean progresivamente incluidos en el proceso expansivo de la matrícula a gran escala que estaba teniendo lugar en el país (primero de manera espontánea para luego ser modelado a través del financiamiento estatal). Muy pronto, calidad y equidad se convertirán en los ejes de la acción sectorial con la aprobación del Programa MECESUP (Mejoramiento de la Calidad y la Equidad en la Educación Superior), que permitirá poner en marcha programas pilotos de acreditación y dar forma a los sistemas públicos de información sectorial, además de financiar numerosos proyectos universitarios (de infraestructura y equipamiento, de desarrollo del doctorado y de fortalecimiento del pregrado y los claustros académicos) a través del Fondo Competitivo, el Fondo de Innovación Académica y los convenios de desempeño. También facilitará el desarrollo de una serie de instancias (como el 
Departamento de Financiamiento Institucional de la Subsecretaría de Educación Superior y la Comisión Nacional de Acreditación) que pasarán a ser piezas clave de la insfraestructura regulatoria del sector. En paralelo, se desarrolla un nuevo sistema de crédito estudiantil (Crédito con Aval del Estado, CAE), disponible para todas las instituciones de educación acreditadas, lo que contribuye tanto al desarrollo del sector privado como de la competencia entre universidades por estudiantes.

La agenda que encarna el Programa de Mejoramiento de la Calidad y Equidad de la Educación (MECESUP) supone una dirección predefinida para el fortalecimiento de la Educación Superior chilena. Aunque el proyecto emerge originalmente de iniciativas realizadas en Argentina y Colombia, se trata básicamente de un experimento político a través del cual el Banco Mundial utiliza a Chile -de modo permanente hasta hoy- como un laboratorio para la forja de una nueva agenda internacional para modernización de la Educación Superior de aquellos estados que aspiran a avanzar hacia su industrialización (WORLD BANK, 2017). El propósito de esta agenda apunta a convertir a las universidades en un importante polo de desarrollo de la economía, asumiendo que el planeta avanza inexorable hacia la consolidación de sociedades del conocimiento, caracterizadas por una fuerza laboral altamente calificada, capaz no sólo de aumentar su productividad, sino también de crear nueva riqueza a través del desarrollo del conocimiento y la innovación. Desde ahora, ésta se entenderá como su principal contribución al bien común.

Estas ideas están estrechamente asociadas a la Escuela de la Nueva Gestión Pública que - en una mirada comparada - ha tenido un acelerado desarrollo en décadas recientes. Como destaca Neave (2012), ella busca que las agencias del Estado (incluyendo las universidades) vayan siendo progresivamente sometidas a la disciplina del mercado y la competencia, respondiendo a incentivos y desincentivos. Esto implica un importante cambio del tipo de relación que los estados han mantenido con sus universidades. Las universidades ganan en autonomía (especialmente en su organización interna, el diseño de los procesos que conducen y la definición de cómo alcanzarán los resultados esperados), pero se expanden sus obligaciones de rendir cuentas frente al estado y la sociedad, lo que implica una creciente cuantificación y especificación de su quehacer y la definición de metas (generalmente asociadas a indicadores de desempeño en aspectos clave). A su vez, los gobiernos dejan de ser responsables del desarrollo de la Educación Superior. En lo que sigue, entregarán financiamiento a las universidades y las someterán a evaluaciones expost como la acreditación, para determinar su capacidad para autogobernarse y responder a los desafíos que enfrentan las sociedades contemporáneas; pero ya no tendrán el deber de aprobar anticipadamente las acciones que las universidades realizan ni ajustar 
su contribución al sector en función de las necesidades de éstas (pues emergen de decisiones autónomas que el Estado no ha tomado).

Los gobiernos y las agencias internacionales han tendido a asumir acríticamente la agenda de la Nueva Gestión Pública. La literatura especializada, en cambio, identifica áreas de preocupación en relación con estos desarrollos (FERLIE; MUSSELIN; ANDRESANI, 2008). Así, se observa que ella posee una orientación economicista que asume que los actores dentro de la Educación Superior son maximizadores de utilidad que operan a través del cálculo racional y en función de resultados cuantitativos. Eso deja fuera de juego a la intuición, la creatividad, la cooperación y el altruismo, aspectos que tienden a definir el trabajo académico en muchos países. Al mismo tiempo, busca disciplinar a las universidades dentro de una lógica que les resulta ajena: es difícil entender sus principales funciones (la enseñanza y la investigación) como procesos productivos que pueden ser modelados y estandarizados. Los principales "clientes" del servicio universitario (los estudiantes) son sus coproductores: ellos deben trabajar activamente para que su formación ocurra y puedan acceder a los diplomas que la Educación Superior entrega y que luego sirven como credenciales laborales. En cuanto instituciones de base pesada, las universidades poseen una limitada capacidad para modelar y dirigir en detalle el trabajo de los profesores universitarios, que además han sido tradicionalmente protegidos por la libertad académica. Al tratar de reducir el quehacer universitario a lo que podría ser su dimensión puramente económica, la Nueva Gestión Pública se queda sin una buena teoría que sea capaz de explicar qué es la Educación Superior, cómo opera en la práctica y el valor que las sociedades le asignan, lo que explica que las reformas a la Educación Superior tienden a producir resultados inesperados (CERYCH; SABATIER, 1986).

Pero esta política, originada en el Programa MECESUP y luego desarrollada más ampliamente, no sólo está afecta a esta línea de objeciones. También existen importantes críticas a su implementación. Los problemas de coordinación entre sus líneas, y especialmente entre aquellas dedicadas a la promoción de la calidad (fondos competitivos) y el control de la calidad (acreditación), facilitan que el proyecto fuera perdiendo su coherencia inicial. Es decir, que la Nueva Gestión Pública ni siquiera fuera capaz de lograr sus propios objetivos. Los diagnósticos producidos a través de la acreditación son escasamente utilizados para el desarrollo de proyectos de mejora de la calidad que el mismo programa financia (SALAZAR; RIFO, 2020).

Adicionalmente, los proyectos que la iniciativa financia no sólo apuntan a resolver los principales problemas que enfrentan las universidades para desarrollar todo su potencial. La naturaleza competitiva de los fondos asociados al MECESUP, y otros programas luego inspirados bajo estas premisas, causa 
que el Estado seleccione las iniciativas que le parecen más promisorias (en los términos definidos en cada convocatoria), pero sin un marco estratégico explícito que vaya orquestando un desarrollo institucional integrado, en función de la trayectoria y situación de cada universidad. Muchas veces, se trata de acciones focalizadas y episódicas que se asocian al despliegue de los distintos grupos de poder dentro de las universidades, reflejando la ausencia de diagnósticos institucionales compartidos (entre las universidades y el gobierno) que permita la progresiva superación de los principales desafíos universitarios. La naturaleza competitiva de sus fondos de desarrollo, por su parte, supone que todas las universidades poseen capacidad y ambición para desarrollar y articular sus propuestas coherentemente. Al dejar tales decisiones en manos de los gestores universitarios, se limita la capacidad del Estado para contribuir consistentemente a superar los principales desafíos que enfrentaban las universidades. Al mismo tiempo, la ejecución de los proyectos financiados se somete a la lógica burocrática de la administración central del Estado, donde la evaluación progresiva del cumplimiento de los objetivos propuesto tiene más que ver con aspectos formales de los procesos que con las finalidades que ellos persiguen.

La pérdida de legitimidad social que afecta a la agenda de políticas inspiradas en el MECESUP tarda en manifestarse y sólo explota a contar de 2011, cuando se vuelve evidente para muchos chilenos que la Educación Superior no se acerca a cumplir las promesas asociadas a su rápida masificación. La acreditación - instrumento dirigido principalmente a regular al emergente sector privado y la expansión de la matrícula, dada la función que cumple regulando el acceso al CAE - pierde su aura tecnocrática al asociarse a una emergente corrupción. Además de revelar que grupos de interés dentro del sector buscan activamente instrumentalizar las políticas en su beneficio propio, también da cuenta que los equipos profesionales que los gobiernos fueron entrenando para la implementación de las políticas de Educación Superior originadas en el Programa MECESUP ofrecen una modesta capacidad para resolver estos problemas una vez que la arquitectura política sectorial entra en crisis.

A propósito de su vinculación fáctica con actores privados, grupos empresariales y tecnocráticos dentro del aparato estatal, muchos de estos actores -burócratas y tecno-burócratas de estos programas e instancias técnicastodavía consideran viable un rápido desarrollo en Chile de una economía del conocimiento sin prestar mucha atención a su evidente inviabilidad dentro del mediano plazo (el foco de estas políticas estuvo en el desarrollo de capacidades dentro de la Educación Superior para acomodar su expansión y no en la articulación entre ésta y la economía). El sello ideológico de la iniciativa también queda en un discreto segundo plano, pues en la mirada de los operadores del 
sistema político, ella es observada como una agenda modernizadora, aunque haya demostrado no producir modernización alguna salvo la expansión de matrícula.

El gran problema del Estado chileno es definir, entonces, la dirección del cambio que debe introducir en la arquitectura política de la Educación Superior a raíz de estos problemas y la crítica del movimiento de 2011. No obstante, enfrenta importantes limitaciones para avanzar en esa dirección. Quizás la más importante sea la falta de una marco conceptual e institucional que permita fundar y organizar la reforma que tanto el gobierno de Bachelet como la sociedad aspiran. Por una parte, esta dificultad no se haya en la ausencia de marcos alternativos - que abundan en la literatura especializada (SCOTT; GALLACHER; PARRY, 2017) - sino en los equipos técnicos en los que el gobierno deposita su confianza. Ellos provienen de los mismos cuadros técnico-burocráticos que se consolidaron (tanto en el gobierno como en las universidades modernizadas) con la implementación del Programa MECESUP y políticas posteriores inspiradas en su diseño. Aunque poseen una escasa visión de conjunto y una limitada legitimidad social para impulsar las bases de una nueva reforma universitaria, tienen la confianza de los principales operadores del sistema político y de distintos intereses sectoriales. Pasarán más de tres años antes de que ellos decanten una propuesta integral que el parlamento pueda discutir. Por eso, la gran transformación de las políticas de Educación Superior vendrá de otro lugar: los economistas del Ministerio de Hacienda. En 2015, se incluye en el presupuesto del estado una asignación especial para financiar los estudios de pregrado que se sobrepone a los sistemas de ayudas estudiantiles: nace la gratuidad universitaria. En este sentido, la dificultad para replantear el modelo neoliberal de Educación Superior radica también en los límites del estado neoliberal mismo, con su centro de poder radicado en la dirección económica (Ministerio de Hacienda), a la que los intereses dominantes tienen acceso, y con un control del tipo Nueva Gestión Pública -como se apunta arriba- o bien propio del Estado subsidiario sobre el desarrollo de sus funciones socio-culturales.

Aunque la política de gratuidad estaba llamada a reorientar fundamentalmente el financiamiento de la Educación Superior, importantes inconvenientes emergen durante su diseño (que sólo se concluye en 2018). Dado su origen intelectual fue concebida como un voucher que se asigna a una persona determinada. No forma parte del financiamiento que se entrega directamente a las universidades, desechando otras opciones como las que se usan en muchos países. Son las personas (y no las universidades) quienes tienen derecho a la gratuidad. Pero no todas las personas son elegibles ni ellas poseen un derecho permanente a su acceso al pregrado. Previniendo la tradicional crítica que se hace en los países en que existe gratuidad (en Argentina, Brasil y Uruguay, ella ha tendido a beneficiar a los estudiantes más ricos, especialmente en las carreras o 
programas más selectivos) se fija un criterio socioeconómico: sólo los estudiantes más pobres tendrán acceso a la gratuidad. Adicionalmente, ellas sólo podrán ejercer tal beneficio individual durante un periodo igual a la duración teórica de su carrera o programa, de manera de asegurar que la gratuidad no sea una excusa para una falta de compromiso individual con los estudios - conducente a mayor ineficiencia dentro del sistema. Adicionalmente, la determinación de las instituciones en que esas personas pueden matricularse también representó un obstáculo importante, ante la ausencia de un criterio objetivo que permitiera diferenciar las universidades razonables de otros proveedores de naturaleza comercial. La ausencia de un criterio más sustantivo, depositó la solución en una fórmula de naturaleza formal (fijado en cuatro años de acreditación institucional) que permite que esta línea de financiamiento se extendiera a las universidades privadas, incluidas las que persiguen finalidades lucrativas.

La reforma integral aprobada en 2018 está informada por los mismos principios. Quizás por eso tuvo una aprobación casi unánime a pesar de las significativas diferencias ideológicas expuestas durante su discusión parlamentaria. A pesar que contiene un conjunto significativo de declaraciones de principios, lo cierto es que mucho de su contenido sólo será definido en la implementación por los mismos equipos tecno-burocráticos. Ostensiblemente, la reforma evita pronunciarse sobre las características que deberá alcanzar el sector a propósito de la reforma y la manera que se asegurará el logro de esos objetivos. Mas bien, transita desde la enunciación de ideas generales a procedimientos y atribuciones, muchas veces con importantes errores que significarán un obstáculo adicional para su puesta en marcha. No es muy difícil imaginar que las ideas que del Programa MECESUP seguirán guiando desarrollo de la Educación Superior, con independencia de lo que opine la sociedad chilena, especialmente a partir del fortalecimiento de la institucionalidad tecnocrática. En suma, la reforma evita hacerse cargo de los principales problemas y desafíos que ella presenta. Sólo durante los próximos años podremos saber si fue una decisión sabia. Mientras tanto, las universidades chilenas seguirán buscando su destino a tientas.

\section{Discusión}

Como vimos, la Educación Superior neoliberal resistió y procesó las críticas del movimiento social precisamente expandiendo el Estado tanto en un sentido financiero como burocrático. Lo que terminó consolidando y blindando la Educación Superior construida en las décadas previas, en lugar de ofrecer 
posibilidades para resolver los problemas derivados de la trayectoria que había recorrido hasta acá.

Es muy probable que ello pueda ser explicado por los poderes que sostienen esa agenda. La determinación fundamental sobre la Educación Superior, entonces, sigue anclada en los poderes que controlan sus dos grandes espacios de reciente creación: las nuevas instituciones elitarias, que forman la élite neoliberal y conservadora bajo un fuerte control ideológico y exclusividad social (sin mayor preocupación por la investigación ni el desarrollo disciplinar); y las nuevas instituciones masivas, que acompasan con finalidades lucrativas un proceso rampante de credencialización en la sociedad que no despunta ni en mayor capital humano ni en ninguna de las promesas de industrialización hechas por el Banco Mundial, ideólogo de las reformas neoliberales de segunda generación. Estos espacios son los que llevan la tendencia de desarrollo en desmedro del sistema público y tradicional, que irónicamente es el que todavía aporta con el grueso de la investigación y el desarrollo intelectual del país.

Estos poderes económicos e ideológicos -partidos de derecha, grupos empresariales, trasnacionales y órdenes ultraconservadoras de la Iglesia Católica- perviven como determinantes en la medida que establecen los límites y se proyectan como la capacidad de presión más efectiva sobre una burocracia estatal que, larvariamente y apuntalada por el Programa MECESUP y sus políticas sucesivas influenciadas por el Banco Mundial, ha terminado por monopolizar la acción estatal para con la Educación Superior, naturalizando su agenda. Los poderes empresariales e ideológicos manipulan la burocracia, primero amparadas en el principio de subsidiariedad del Estado, que les permite reclamar un financiamiento equivalente al de la educación pública, y luego, estableciendo vínculos orgánicos con ella, los que llegan incluso a la corrupción formalmente establecida por tribunales de justicia. Es así como tanto en un sentido conceptual como práctico no hay espacio para la educación pública; toda institución educativa -sea privada o estatal- queda entonces puesta como una instancia genérica con la que el Estado se relaciona en base a preceptos burocráticos y estandarizados de calidad y equidad. Se anula el espacio para la educación pública al mismo tiempo que la burocracia reclama el interés general y la razón técnica, ocultando de tal modo su sometimiento a los intereses empresariales e ideológicos particulares que orgánicamente la dirigen.

Más allá de las críticas que puedan elaborarse contra el economicismo extremo de esta agenda, de suyo incompatible con un enfoque de derechos, lo cierto es que fracasa en sus propios objetivos. Sus indicadores y mediciones de productividad comienzan a ser vistas como fines en sí mismos (sin importar que ellos sean excesivamente formales, conservadores y anclados en una mirada economicista de la Educación Superior que se niega a reconocer las 
revolucionarias transformaciones que el sector experimentó desde la puesta en marcha de la agenda MECESUP), instaurando un comportamiento instrumental en los actores que desnaturaliza el propio sentido de cada uno de estos instrumentos de política. A la larga, los mismos sujetos han tendido a internalizar estas aproximaciones, lo que va desde una juventud que reclama a la Educación Superior combinando planteamientos antineoliberales y derechos que sienten que merecen en su calidad de clientes, y académicos que se orientan por la adquisición formal de logros (publicaciones indexadas, fondos adjudicados, etc.) más que por proyectos sustantivos de índole científico e intelectual.

Si algo ha hecho con éxito esta tecno-burocracia, es desplazar, por la vía de la tecnocratización de los debates, a otros intereses sociales de la determinación sobre la Educación Superior. De tal modo, vinculada orgánicamente con los grandes poderes de la Educación Superior, y con autonomía relevante de las autoridades estatales electas, deviene más bien una fuerza de administración y de blindaje político que un núcleo intelectual capaz de elaborar una agenda con sentido. El peso de la inercia los lleva a naturalizar los abordajes dados, incluso aunque estos fallen en una serie de planos. De ahí que en los sucesos de la Reforma de 2014 a 2016, bajo mandato de Bachelet, se combine tanto esta naturalización ideológica como la presión de los poderes empresariales e ideológicos en el resultado final.

Irónicamente, las afirmaciones de Bardón que vimos al inicio de este artículo terminan proyectándose y materializándose en los gobiernos civiles, incluso bajo retóricas progresistas o de centro-izquierda. Más paradojal todavía es que estas máximas economicistas no apuntalan un mercado competitivo, sino a una burocracia que se parece más a las castas tecno-burocráticas de los socialismos reales estalinistas que a los mundos imaginados por Hayek o Friedman. Es que, como dijimos al inicio, el neoliberalismo no es un proyecto que tenga que ver con el empequeñecimiento del Estado, sino todo lo contrario, se trata de un proyecto que requiere y usa el Estado para construir, proteger y blindar los mercados de cualquier control democrático posible.

\section{REFERENCIAS}

BACHELET, Michele. Programa de gobierno, 2014-2018, 2013.

BRUNNER, José Joaquín. Tendencias de cambio en el sistema de educación superior chileno: 1973-1982. Santiago: FLACSO, 1982. 
BRUNNER, José Joaquín. El movimiento estudiantil ha muerto: nacen los movimientos estudiantiles. Santiago: FLACSO, 1985.

BRUNNER, José Joaquín. Educación superior en Chile: entre el Estado, el mercado y los intereses académicos. Santiago: FLACSO, 1987.

CERYCH, Ladislav; SABATIER, Paul. A. Great expectations and mixed performance: The implementation of higher education reforms in Europe. London, Trentham Books, 1986.

CONFECH (Confederación de Estudiantes de Chile). Bases para un acuerdo social por la educación chilena, 2011. Recuperado 25 de mayo de 2020. http://www.archivochile. com/Chile_actual/01_mse/1/MSE1_0013.pdf.

DONOSO, Sofia. Dynamics of change in Chile: Explaining the emergence of the 2006 Pingüino movement. Journal of Latin American Studies, Cambridge, UK, v. 45, n. 1, p. $1-29,2013$.

FERLIE, Ewan; MUSSELIN, Christine; ANDRESANI, Gianluca. The steering of higher education systems: A public management perspective. Higher Education, Amsterdam, v. 56, n. 3, p. 325-348, 2008.

FLEET, Nicolas; GUZMÁN-CONCHA, César. Mass Higher Education and the 2011 Student Movement: Material and Ideological Implications. Bulletin of Latin American Research, Oxford, UK and Malden, USA, v. 36, n. 2, p. 160-176, 2017.

FLEET, Nicolas. Mass intellectuality of the neoliberal state: mass higher education, public professionalism and state effects in Chile. 2019. Tesis (Doctoral Sociology) University of Cambridge, Cambridge, UK, 2019.

GUAJARDO, Fabián; MIRANDA, Camila; SANHUEZA, José Miguel ¿Por qué la reforma educacional está al borde del fracaso? Balance y proyecciones para las fuerzas de cambio. Cuadernos de Coyuntura Fundación Nodo XXI, Santiago, v. 5, n. 16, p. 23-33, 2017.

GUTIÉRREZ, Claudio; LÓPEZ, Mercedes; RUÍZ SCHNEIDER, Carlos. Educación superior y segregación social en Chile. Historia de sus ideas, políticas e instituciones (apuntes de clase). Santiago: CEIBO, 2018.

MONCKEBERG, Maria Olivia. La privatización de las universidades: una historia de dinero, poder e influencias. Santiago: Copa Rota, 2005.

MONCKEBERG, Maria Olivia. El negocio de las universidades en Chile. Santiago: Random House, 2007.

NEAVE, Guy. The evaluative state, institutional autonomy and re-engineering higher education in Western Europe: The prince and his pleasure. Doordrecht: Springer, 2012. NÚCLEO SOCIOLOGÍA CONTINGENTE. Informe de Resultados Oficial Encuesta Zona Cero. Santiago: Universidad de Chile, 2020. 
O'DONNELL, Guillermo. Reflections on the Patters of Change in the BureaucraticAuthoritarian State. Latin American Research Review, [s.l. ], v. 13, n. 1, p. 3-38, 1978.

ORELLANA, Víctor. Nuevos estudiantes y tendencias emergentes en la educación superior. Una mirada al Chile del mañana. En Nueva geografía de la educación superior y de los estudiantes. Una cartografia del sistema chileno, su actual alumnado y sus principales tendencias. Santiago: Ediciones San Sebastián, 2011. p. 79-142.

ORELLANA, Víctor. Sobre el malestar social con la educación y la energía del movimiento social. El primer paso del Chile del siglo XXI. En Es la Educación, Estúpido. Santiago: Planeta, 2012.

RUIZ ENCINA, Carlos. Conflicto social en el "neoliberalismo avanzado": Análisis de clase de la revuelta estudiantil en Chile. CLACSO: Buenos Aires, 2013.

RUÍZ SCHNEIDER, Carlos. Lo Público y lo Privado en la Educación Chilena. En Grafias Filosóficas. Santiago: Universidad de Chile, 2007.

SALAZAR, José Miguel; RIFO, Mauricio. Bajo el Péndulo de la Historia: Cinco décadas de masificación y reforma en la educación superior chilena (1967-2019). Economía y Política, Santiago; Viña del Mar, v. 7, p. 27-61, 2020.

SALAZAR, José Miguel; LEIHY, Peodair S.. El Manual Invisible: Tres décadas de políticas de educación superior en Chile (1980-2010). Education Policy Analysis Archives, Arizona, USA, v. 21, n. 34, p. 1-35, 2013.

SCOTT, Peter; GALLACHER, Jim; PARRY, Gareth. (ed.). New languages and landscapes of higher education. Oxford University Press, 2017

SIES (Servicio de Información de Educación Superior). Informe Personal Académico en Educación Superior 2019. Santiago, Ministerio de Educación, 2019.

SILVA, Eduardo. The State and Capital in Chile: Business Elites, Technocrats, and Market Economics. Boulder, CO: Westview Press, 1996.

SIMBÜRGER, Elisabeth. Moving through the city: visual discourses of upward social mobility in higher education advertisements on public transport in Santiago de Chile. Visual Studies, [s.l.], v. 28, n. 1, p. 67-77, 2013.

TORCHE, Florencia; WORMALD, Guillermo. Estratificación y movilidad social en Chile: entre la adscripción y el logro.Santiago: CEPAL, 2004.

TROW, Martin Reflection on the Transition from Elite to Mass to Universal Access: Forms and Phases of Higher Education in Modern Societies Since WWII. En: International Handbook of Higher Education. Dordrecht: Springer, 2007. p. 243-280.

VERCELLONE, Carlo. From Formal Subsumption to General Intellect: Elements for a Marxist Reading of the Thesis of Cognitive Capitalism. Historical Materialism, [s.l.], v. 15, n. 1, p. 13-36, 2007. 
OYARCE, N. G. F.; LEIHY, P. S.; ZEGERS, J. M. S. Crisis de la Educación Superior en el Chile...

WORLD BANK. Higher Education for Development. An Evaluation of the World Bank Group's Support.Washington, D.C.: Inter-American Development Bank, 2017.

Texto recibido en $02 / 08 / 2020$.

Texto aprobado en 20/09/2020. 